\title{
What's New in Narcolepsy? Review of All Published Literature in 2020
}

\section{Rajib Dutta*}

MD Neurology, India

*Corresponding Author: Rajib Dutta, MD Neurology, India.

E-mail: rajibdutta808@gmail.com

ORCID ID: http://orcid.org/0000-0002-6129-1038

DOI: 10.31080/ASNE.2020.03.0162
Received: February 24, 2020

Published: February 28, 2020

(C) All rights are reserved by Rajib Dutta.

\begin{abstract}
Narcolepsy is a neurological disorder that affects ability to wake and sleep. Individuals with narcolepsy have excessive, uncontrollable daytime sleepiness, cataplexy, hallucinations, sleep paralysis etc. It is divided into type 1 and 2 on the basis of cataplexy. Type 1 is autoimmune. Recently lot of research and new case reports has been published because of the association with impaired cognitive ability, poor quality of life, and risk of work, home, and car accidents. It can affect children, adolescents and even elderly. FDA and EMA approved drugs are available. In this article all published literatures of 2020 regarding narcolepsy are reviewed.
\end{abstract}

Keywords: Narcolepsy; 2020

\section{Abbreviations}

HLA: Human Leukocyte Antigen; IH: Idiopathic Hypersomnia; OSA: Obstructive Sleep Apnea; ISS :Insufficient Sleep Syndrome; REM: Rapid Eye Movement; NREM: Non Rapid Eye Movement; COPD: Chronic Obstructive Pulmonary Disease; ESKD: End Stage Kidney Disease; AED: Anti-Epileptic Drug; DNMT: DNA Methyltransferase; SOREMP: Sleep-Onset REM Periods; CSF: Cerebrospinal Fluid; HRQOL: Health Related Quality Of Life; PWN: Person With Narcolepsy; PSG: Polysonogram; PAX: Paired Box Protein; ADHD: Attention Deficit Hyperactivity Disorder, RCT: Randomized Controlled Trial; TIA: Transient Ischemic Attack

\section{Introduction}

Narcolepsy is a chronic sleep disorder which is very disabling to human beings. Currently it is divided into two subtypes Narcolepsy type 1 (NT1) and Narcolepsy type 2 (NT2). NT1 presents with excessive daytime sleepiness (EDS) and cataplexy whereas NT2 does not have cataplexy [1-4]. Individuals who suffer from narcolepsy also have symptoms like automatic behavior, memory complaints, and dream delusions which are often underreported. Patients with narcolepsy are also prone to develop fatiguability, obesity and psychiatric manifestations like depression, anxiety and eating disorders and severe negative impact on daily functioning and quality of life.NT1is due to loss of hypocretin (also called orexin) cells in the hypothalamus.NT2 on the other hand comprise of EDS and abnormal expressions of REM sleep on the multiple sleep latency test (MSLT) [5]. Other causes may be familial or medical conditions like tumor in the hypothalamic region in brain, paraneoplastic syndrome or storage diseases like Niemann-Pick disease type $\mathrm{C}$.

Narcolepsy with cataplexy has an estimated prevalence of 25 - 50 per 100000 . The incidence is estimated to be $0.74-1.37$ per 100000 person-years [6]. There is no reliable prevalence or incidence estimations of narcolepsy without cataplexy at this moment. Age of onset is usually between 15 - 35 years, but may start at any age. EDS is the first symptom to occur in majority of patients [7]. NT1 may be autoimmune in nature linked to allele HLA DQB1*06:02 [8] and CD8+ T cells may be responsible in neuronal damage seen in human narcolepsy [14,25]. Cataplexy must be differentiated from cataplexy-like conditions in normal subjects like laugh and drop attacks. Medical conditions like transient ischemic attack, seizures, psychogenic attacks should be kept in mind while diagnosing narcolepy. Individuals with shiftwork 
disorder, sleep deprivation, and sleep apnea can have EDS and same MSLT findings [9]. Infection with H1N1 or other strains $[10,13]$ and S. pyogenes [11] has shown positive correlation.Antitribbles homolog 2 autoantibodies has reported in narcolepsy, may be secondary to hypocretin cell loss [12].

\section{Current updates}

Brennan., et al. reported a case where T-cell-mediated neuronal destruction from hypothalamus to brain stem was found in a patient post mortem presented with limbic encephalitis, parkinsonism features and narcolepsy in endometrial carcinoma suspected as a paraneoplastic syndrome with negative antibodies. Novel antibodies related to paraneoplastic syndromes should be studied or reported and can not be ruled out as suggested by authors [15]. Naeimi., et al. (year) reported about the connection of neurodegeneration, REM sleep behavior disorder related to narcolepsy [16]. Zaidi., et al. in their crossectional observation study found narcolepsy along with sleep apnoea and insomnia were more prevalent among COPD patients from lower socioeconomic status [17]. Schinkelshoek., et al. in crossectional observation studied about daytime sleep state misperception with EDS with routine MSLT. They reported it is common in people with NT1, NT2, IH, OSA, and ISS and is more frequent in patients with longer sleep onset latencies who only reach non-REM sleep stage 1 during a nap [18].

Chen., et al. examined the association of anxiety disorders and narcolepsy in population-based, retrospective case-control study and reported two important findings 1)higher comorbidity rates of anxiety disorders in narcoleptic patients in age group 12 - 17 years 2)females outnumbered males [19]. Lind., et al. screened for autoantibody targets in post-vaccination narcolepsy using proteome arrays however warranted requirement of further studies to identify hypothetical autoantigens related to the pathogenesis of Pandemrix ${ }^{\circledR}$-induced NT1 [20]. Xiao., et al. in their case-control study reported alterations in brain network connectivity and regional topological properties in narcoleptic adolescents and positive association with their sleepiness, depressive feelings and impulsive behaviors [21]. Franceschini., et al. studied narrative recalls already diagnosed as NT1,three clusters was studies a) situations that trigger attacks of cataplexy b) bodily sensations during attacks c) control strategies during episodes. They reported patients disregarded emotional experiences thus affecting the ability to name, recognize and regulate critical emotions [22]. Dauvilliers., et al. reported NSS(Narcolepsy Severity Scale) as a severity and consequences of the five major narcolepsy symptoms: daytime sleepiness, cataplexy, hallucinations, sleep paralysis, and disturbed nighttime sleep (DNS) is a valid one responsive to treatment which can be used in clinics as well as research [23]. Prevalence of narcolepsy in patients with ESKD is not well characterized but has been reported to be around $1.4 \%$ as reported in literature. Narcolepsy should be in differential diagnosis in patients with sleepiness/hypersomnolence in patients with ESKD [24]. Symptomatic narcolepsy/cataplexy can occur in dogs and can be secondary to brainstem encephalitis along with cardiovascular changes. The case as reported by Mari and Shea involves a 4 yr old, intact female cocker spaniel diagnosed with meningoencephalitis of unknown origin affecting the mesencephalon, pons and rostral medulla oblongata supported by MRI and CSF findings. Immunosuppressive treatment with prednisolone and cytosine arabinoside initially and subsequent switching to cyclosporine led to complete recovery [26].

Schneider., et al. recently reported a case of a female patient with loss of consciousness and generalized muscle atonia with no loss of anal or urinary sphincter tone and preservation of eye movements, initially treated as a seizure disorder, no response to anti seizure medications was found to have a laughter related facial cataplexy. This clinical findings can be missed by inexperienced clinicians in a diagnosable narcolepsy case when presents acutely with medication refractory seizures including multiple AEDs [27]. Mutations in exons 21 and 20 of the DNMT1 gene have been associated with ADCADN (Autosomal Dominant Cerebellar Ataxia with Deafness and Narcolepsy), Postiglione., et al. in a case reported about a 44-year-old female with personal and familiar longstanding history of progressive bilateral sensorineural deafness, and sensitive cerebellar ataxia, presenting with brief episodes of falls while laughing and excessive diurnal somnolence. Genetic testing found p.Glu575Lys mutation in exon 21 in the proband and her mother. However after 4 years of follow-up her walking ability declined, hypersomnolence, repeated PSG documented REM sleep latency shortening, and evidence of de novo spontaneous SOREMPs, although normal CSF hrct-1(hypocretin) at first and second evaluation. Classical diagnosis of NT1 was never made but course of the disease was progressive [28].

Berntsson., et al. reported first case of a patient with complex sleep disorder, PAX6 haploinsufficiency, aniridia and pineal gland hypoplasia diagnosed with narcolepsy type1with pathogenic c.959-1G>A mutation in the PAX6 gene in all 4 members of the 
family suggesting penetrance [29]. Ong., et al. studied about quality of life in patients with narcolepsy. Patient-Reported Outcomes Measurement Information System (PROMIS) measures appeared to be suitable and efficient instruments for assessing HRQoL in PWN [30]. Chang., et al. in their case control study reported significantly increased risk of narcolepsy patients to burn injury compared to unaffected controls. Use of stimulants was associated with a lower incidence rate of developing burn injury [31]. Although loss of hypocretin/Orexin (Hcrt/Ox) peptides or their receptors have been associated with the disease, a circuit perspective of the pathophysiological mechanisms of the narcolepsy symptoms that encompasses brain regions, neuronal circuits, cell types, and transmitters beyond the Hcrt/Ox system was proposed by Adamantidis., et al [32].

Narcolepsy monitor app is reported to be a helpful tool to gain more insight into the individual burden of narcolepsy symptoms over time and may serve as a patient-reported outcome measure [33]. Scott., et al. in their recent case control comparative study showed several sleep related problems are common in inflammatory bowel disease(IBD) including narcolepsy. IBD group reported significantly more severe experiences of five of the seven domains of the sleep-50 [34]. Pediatric patients with narcolepsy had greater comorbidity, higher health care utilization, and higher costs than patients without narcolepsy [35].

Pitolisant, orally available selective histamine $\mathrm{H} 3$ receptor inverse agonist recently approved for NT1 and NT2 and EDS can be used when coexistent Parkinson's disease is present [36]. Pitolisant administered at up to the recommended maximum dose of $36 \mathrm{mg}$ once daily reduces EDS and cataplexy in adults with narcolepsy relative to placebo. Most common treatment-emergent adverse events included headache, insomnia and anxiety otherwise it is well tolerated. It is of similar efficacy as Modafinil in management of EDS. It has minimal abuse potential, once-daily administration is for treatment of NT1/2 is an attractive option [37,42]. Children body weight $<40 \mathrm{~kg}$ doses up to $17.8 \mathrm{mg} / \mathrm{d}$ is tolerable and $>40 \mathrm{~kg} 35.6$ $\mathrm{mg}$ [38]. Immunotherapies include intravenous immunoglobulin (IVig), followed by corticosteroids, plasmapheresis, and monoclonal antibodies which can be used in patients with NT1, however improvement of symptoms is particularly seen when patients were treated close to disease onset. Inadequate timing of treatment, placebo effects, and spontaneous improvement due to the natural disease course can account for contrasting findings in the study of Giannoccaro., et al [39]. Several medications still in development (FT218, JZP-258, AXS12, THN102, SUVN-G3031, TAK925), provide new options for the treatment of narcolepsy which need to be studied [40]. Modafinil is a nonamphetamine nootropic drug with an increasingly therapeutic interest due to its different sites of action and behavioral effects in comparison to cocaine or amphetamine in narcolepsy is studied. Most common adverse effects are headache, insomnia, anxiety, diarrhea, dry mouth and rise in blood pressure and heart rate. Infrequently, severe dermatologic effects in children, including maculopapular and morbilliform rash, erythema multiforme and Stevens-Johnson Syndrome have been reported. Intoxication and dependence associated to modafinil are uncommon [41]. Immediate-release amphetamine medication and sustained-release amphetamine medication are used to treat ADHD and narcolepsy in both adult and pediatric population [43].

\section{Discussion}

Narcolepsy is a disabling neurological disorder which when untreated can be fatal. Various comorbid conditions can coexist like cardiometabolic risk factors, neuropsychiatric conditions(mood disorders, anxiety) and sleep disorders such as restless legs syndrome, periodic nocturnal leg movements, rapid eye movement (REM) and non-REM (NREM) sleep parasomnias etc.NT1 can be due to irreversible destruction of hypocretin/orexin (ORX) neurons with an absence or low levels of ORX in the cerebrospinal fluid (CSF), probably by an autoimmune mechanism, in genetically predisposed individuals which is linked to allele HLA DQB1*06:02. NT2 is not studied in deep till date and further research is warranted. FDA and EMA approved drugs are available in market targeting the disease at the very onset.

\section{Conclusion}

Published studies showed encouraging results particularly regarding improvement of EDS, but also, to a lesser extent, regarding improvement of cataplexy. The review has highlighted some novel case reports in which meningoencephalitis or other paraneoplastic syndromes can present with narcolepsy. Cataplexy sometimes can be confused with seizures, drop or psychogenic attacks and TIA. Immune-based therapies are controversial, and if used should be given close to disease onset. There is no convincing evidence at this moment which can suggest immune modulating therapies improve symptoms or long- term outcome. Potential side effects with immune based therapies is a grave concern because no single immunomodulating therapy is approved. More RCTs are required in future address all this issues. 


\section{Acknowledgement}

The author has no relevant affiliations or financial involvement with any organization or entity with a financial interest in or financialconflict with the subject matter or materials discussed in the manuscript. Special thanks to my supervisor Professor Dr. Huifang Shang who gave initial ideas and supported me through this research study. I would also like to thank Dr. Swatilekha Roy Sarkar for her valuable feedback on the manuscript and literature screening.

\section{Bibliography}

1. American Academy of Sleep Medicine, International classification of sleep disorders, 3rd ed., American Academy of Sleep Medicine (2014).

2. Scammell TE. "Narcolepsy". The New England Journal of Medicine 373 (2015): 2654-2662.

3. Dauvilliers Y., et al. "Narcolepsy with cataplexy". Lancet 369 (2007): 499-511.

4. Overeem S., et al. "Narcolepsy: clinical features, new pathophysiologic insights, and future perspectives". Journal of Clinical Neurophysiology 18 (2001): 78-105.

5. Baumann CR., et al. "Challenges in diagnosing narcolepsy without cataplexy: a consensus statement”. Sleep 37 (2014): 1035-1042.

6. Longstreth WT., et al. "The epidemiology of narcolepsy". Sleep 30 (2007): 13- 26.

7. Luca G., et al. "European Narcolepsy Network. Clinical, polysomnographic and genome- wide association analyses of narcolepsy with cataplexy: a European Narcolepsy Network study". Journal of Sleep Research 22 (2013): 482-495.

8. Bassetti CLA., et al. "Narcolepsy - clinical spectrum, aetiopathophysiology, diagnosis and treatment". Nature Reviews Neurology 15.9 (2019): 519-539.

9. Goldbart A., et al. "Narcolepsy and predictors of positive MSLTs in the Wisconsin Sleep Cohort". Sleep 37 (2014): 1043-1051.

10. Partinen M., et al. "Narcolepsy as an autoimmune disease: the role of H1N1 infection and vaccination". The Lancet Neurology 13 (2014): 600-613.

11. Aran A., et al. "Elevated anti- streptococcal antibodies in patients with recent narcolepsy onset”. Sleep 32 (2009): 979983.
12. Cvetkovic- Lopes V., et al. "Elevated tribbles homolog 2specific antibody levels in narcolepsy patients". Journal of Clinical Investigation 120 (2010): 713-719.

13. Luo F., et al. "Autoimmunity to hypocretin and molecular mimicry to flu in type 1 narcolepsy". Proceedings of the National Academy of Sciences of the United States of America 115 (2018): E12323-E12332.

14. Pedersen NW., et al. "CD8+ T cells from patients with narcolepsy and healthy controls recognize hypocretin neuronspecific antigens (2019): 10,837.

15. Brennan D., et al. "Antibody-Negative Paraneoplastic Limbic Encephalitis, Parkinsonism, Hypothermia, and Narcolepsy Associated with Endometrial Carcinoma". Movement Disorders Clinical Practice7.2 (2020): 223-227.

16. Naeimi SA., et al. "Neurodegeneration in NarcolepsyAssociated Rapid Eye Movement Sleep Behavior Disorder". Movement Disorders Clinical Practice 7.2 (2020): 208-210.

17. Imran Haider Zaidi SM., et al. "Level of sleep disorders among patients with chronic obstructive pulmonary disease". Journal of Pakistan Medical Association 70.2 (2020): 259-263.

18. Schinkelshoek MS., et al. "Daytime sleep state misperception in a tertiary sleep centre population [published online ahead of print, 2020 Jan 10]". Sleep Medicine 69 (2020): 78-84.

19. Chen TY., et al. "Sex and age differences in the association between anxiety disorders and narcolepsy: A nationwide population-based case control study". Journal of Affective Disorders 264 (2020): 130-137.

20. Lind A., et al. "Screening for autoantibody targets in postvaccination narcolepsy using proteome arrays". Scandinavian Journal of Immunology (2020): e12864.

21. Xiao F., et al. "Resting-state brain network topological properties and the correlation with neuropsychological assessment in adolescent narcolepsy". Sleep (2020): 018.

22. Franceschini C., et al. "Giving a voice to Cataplectic experience: recalls from patients with narcolepsy type 1". Journal of Clinical Sleep Medicine (2020): 8286.

23. Dauvilliers Y., et al. "Narcolepsy Severity Scale: A reliable tool assessing symptom severity and consequences”. Sleep (2020): 009.

24. So JY., et al. "A Guide to Management of Sleepiness in ESKD". American Journal of Kidney Diseases (2020) 31066-31062. 
25. Kornum BR and Jennum P. "The case for narcolepsy as an autoimmune disease". Expert Review of Clinical Immunology (2020): 1-3.

26. Mari L and Shea A. "Symptomatic Narcolepsy/Cataplexy in a Dog with Brainstem Meningoencephalitis of Unknown Origin". Journal of the American Animal Hospital Association 56.2 (2020): e56201.

27. Schneider L and Ellenbogen J. "Images: Facial cataplexy with demonstration of persistent eye movements". Journal of Clinical Sleep Medicine 16.1 (2020):157-159.

28. Postiglione E., et al. "Cataplexy and ataxia: red flags for the diagnosis of DNA methyltransferase 1 mutation". Journal of Clinical Sleep Medicine 16.1 (2020):143-147.

29. Berntsson SG., et al. "Aniridia with PAX6 mutations and narcolepsy". Journal of Sleep Research (2020): e12982.

30. Ong JC., et al. "How Does Narcolepsy Impact Health-Related Quality of Life? A Mixed-Methods Study". Behavioral Sleep Medicine (2020): 1-14.

31. Chang ET., et al. "Risk of burn injury in patients with narcolepsy: A nationwide population-based cohort study". Journal of Burn Care and Research (2020): 004.

32. Adamantidis AR., et al. "A circuit perspective on narcolepsy". Sleep (2020): 296.

33. Quaedackers L., et al. "A Mobile App for Longterm Monitoring of Narcolepsy Symptoms: Design, Development, and Evaluation". Journal of Medical Internet Research 8.1 (2020): e14939.

34. Scott AJ., et al. "A comparative study of the nature and magnitude of problems sleeping in inflammatory bowel disease (IBD) compared to healthy controls". Psychology, Health and Medicine (2020): 1-11.

35. Carls G., et al. "Burden of disease in pediatric narcolepsy: a claims-based analysis of health care utilization, costs, and comorbidities". Sleep Medicine 66 (2020): 110-118.

36. Liguori C., et al. "Pitolisant for treating narcolepsy comorbid with Parkinson's disease”. Sleep Medicine 69 (2020): 86-87.

37. Lamb YN. "Pitolisant: A Review in Narcolepsy with or without Cataplexy". CNS Drugs 34.2 (2020): 207-218.
38. Lecendreux M., et al. "Pharmacokinetics of pitolisant in children and adolescents with narcolepsy". Sleep Medicine 66 (2020): 220-226.

39. Giannoccaro MP., et al. "Immunotherapy in Narcolepsy". Current Treatment Options in Neurology 22.1 (2020): 2.

40. Thorpy MJ. "Recently Approved and Upcoming Treatments for Narcolepsy”. CNS Drugs 34.1 (2020): 9-27.

41. Sousa A and Dinis-Oliveira RJ. "Pharmacokinetic and pharmacodynamic of the cognitive enhancer modafinil: Relevant clinical and forensic aspects". Substance Abuse (2020): 1-19.

42. Li S and Yang J. "Pitolisant for treating patients with narcolepsy". Expert Review of Clinical Pharmacology (2020): 1-6.

43. Sharbaf Shoar N., et al. "Dextroamphetamine-Amphetamine". In: Stat Pearls. Treasure Island (FL): StatPearls Publishing (2020).

\section{Assets from publication with us}

- Prompt Acknowledgement after receiving the article

- Thorough Double blinded peer review

- Rapid Publication

- Issue of Publication Certificate

- High visibility of your Published work

Website: https://www.actascientific.com/

Submit Article: https://www.actascientific.com/submission.php

Email us: editor@actascientific.com

Contact us: +919182824667 